\title{
A 2020 Review of Mental Health Comorbidity in Gender Dysphoric and Gender Non-Conforming People
}

\author{
María Paz-Otero, $M D^{1}$, Antonio Becerra-Fernández $M D, P h D^{1,2}$, Gilberto Pérez-López, $M D, P h D^{2,3}$ and \\ Domingo Ly-Pen, MD, PhD $2,4^{*}$ (D) \\ ${ }^{1}$ Department of Biomedical Sciences, University of Alcalá, Madrid, Spain \\ ${ }^{2}$ Charter member of the MUTWOG (Madrid University Transgender Working Group), Spain \\ ${ }^{3}$ Department of Endocrinology and Nutrition, "La Luz" Hospital, Madrid, Spain \\ ${ }^{4}$ Abbey House Medical Centre, Navan, Ireland
}

\begin{abstract}
In the last few years, several studies have suggested the presence of high rates of psychopathology in the trans population and those with gender variability. The purpose of this paper has been to investigate the prevalence of psychiatric disorders in gender dysphoric and gender non-conforming people, as well as their relationship to the stigma and discrimination that they often suffer. For this purpose, this review includes 37 studies published in the last five years, which address the prevalence of various psychiatric pathologies while also addressing possible factors related to them. Among all the psychiatric disorders considered, the most frequent are depression, suicidal ideation, and anxiety. Other pathologies that, although to a lesser extent, also show a higher prevalence than in the cis population, are substance abuse, eating disorders, suicide attempts, autism spectrum disorders or dissociative disorders. Considering the results according to the age of the subjects, adolescence was shown to be a particularly vulnerable period. In addition, through the analysis of multiple circumstances related to transsexuality, it becomes clear how the stress of these minorities justifies the association between transsexuality and psychiatric pathology. In this way, we should start a "depathologization" and we should understand that it is not gender variability that implies the existence of psychopathology in itself. These data point to the need for future research with meticulous methodology.
\end{abstract}

Keywords

Mental health, Gender dysphoria, Transsexuality, Gender nonconforming, Review

\section{Glossary of Terms}

We consider of great importance a brief summary of the main concepts about transsexuality, to understand what we are speaking about [1].

Assigned gender: The initial gender attributed to an individual after birth. For most individuals, this corresponds to the sex on their original birth certificate (aka assigned gender, birth sex).

Cisgender: A term for individuals whose experienced and expressed gender are congruent with their gender assigned at birth (i.e.: not transgender).

Detransition: The cessation or reversal of a transgender identification or gender transition, whether by social, legal, or medical means. It can be temporary or definitive.

Desistance: It is the cessation of transgender identity or gender dysphoria.

Diagnostic and Statistical Manual of Mental Disorders
DSM: Handbook used by health care professionals as the authoritative guide to the diagnosis of mental disorders. DSM contains descriptions, symptoms, and other criteria for diagnosing mental disorders.

Experienced gender: One's sense of belonging or not belonging to a particular gender (aka gender identity).

Expressed gender: How one expresses one has experienced gender.

*Corresponding author: Domingo Ly Pen, Charter member of the MUTWOG (Madrid University Transgender Working Group), Spain; Abbey House Medical Centre, Navan, Co Meath, C15 D290, Ireland, Tel: +3530469051500

Accepted: March 06, 2021

Published online: March 08, 2021

Citation: Paz-Otero $M$, Becerra-Fernández A, Pérez-López G, et al. (2021) A 2020 Review of Mental Health Comorbidity in Gender Dysphoric and Gender Non-Conforming People. J Psychiatry Treat Res 3(1):44-55 
Female to Male (FtM): Transsexual male, a person assigned female at birth, whose gender identity is male.

Gender: a person's social status as male (boy/man) or female (girl/woman), or alternative category.

Gender-affirming surgery: Surgical procedures intended to alter a person's body to affirm their experienced gender identity, aka sex reassignment surgery, gender reassignment surgery, and gender-confirming surgery.

Gender assignment: Assignment of a gender to an individual. In typically developed newborns, the initial gender assignment (aka birth-assigned gender) is usually made based on the appearance of the external genitalia.

Gender binary: A gender-categorization system limited to the two options, male and female. Individuals who identify outside the gender binary may use a variety of gender identity labels, including genderqueer or non-binary.

Gender dysphoria (not capitalized): Distress caused by the discrepancy between one's experienced/expressed gender and one's assigned gender and/or primary or secondary sex characteristics. It is about identity, not sexual orientation.

Gender Dysphoria (GD) (capitalized): A diagnostic category in DSM-5, with specific diagnoses defined by age group-specific sets of criteria.

Gender-fluid: When gender identity changes over time. A gender-fluid person might identify as a woman one day and a man the next. Aka as "agender", "bigender", or another non-binary identity.

Gender identity: One's identity as belonging or not belonging to a particular gender, whether male, female, or a non-binary alternative, aka experienced gender.

Gender Identity Disorder (GID): A diagnostic category in DSM-III and DSM-IV, which was replaced in DSM-5 by GD.

Gender incongruence (not capitalized incongruence between experienced/expressed gender and assigned gender, and/or psychical gender characteristics.

Gender Incongruence (capitalized): A diagnostic category (analogous to GD in DSM-5) proposed for ICD-11.

Gender role: cultural/societal definition of the roles of males and females (or of alternative genders).

Gender variance: Any variation of experienced or expressed gender from socially ascribed norms within the gender binary.

Genderqueer: An identity label used by some individuals whose experienced and/or expressed gender does/do not conform to the male/female binary. Aka as "non-binary".

Internalized transphobia: Fear, hatred, lack of acceptance or discomfort with one's own trans gender identity, as a consequence of the internalization of society's normative idea of gender.

International Classification of Disease (ICD): Diagnostic tool used to classify diseases, injuries, and causes of death.
Intersex conditions: A subset of the somatic conditions known as "disorders of sex development" or "differences of sex development" in which chromosomal sex is inconsistent with genital sex, or in which the genital or gonadal sex is not classifiable as either male or female.

Male to Female (MtF): Transsexual female, a person assigned male at birth, whose gender identity is female.

Minority stress: It refers to high and chronic levels of stress experienced by people who, due to their gender variability, belong to a minority group. It is due to the social and internalized stigma, as well as discrimination and prejudice suffered.

Pathologization: Referring to the perception and approach of transsexuality as if it were a disorder that can be treated through psychiatric procedures.

Sex: A person's categorization as biologically male or female generally based on the genitals and reproductive tract.

Sex assigned at birth: The sex or gender first assigned to an individual after birth. Also known as "natal gender", "birth-assigned sex", and "gender assigned at birth".

Sexual identity: Perception that a person has about him/ herself with respect to his own body based on the evaluation he makes of his/her physical or biological characteristics. They may or may not match the sex assigned at birth.

Sexual orientation: A person's pattern of sexual attraction and physiological arousal to others of the same, other, both, or neither sex. It is independent of gender identity or sexual identity.

Stigma: A condition, attribute, trait or behavior that causes the carrier person to be included in a social category towards whose members a negative response is generated. Because of this, they are often seen as culturally unacceptable or inferior.

Transition: Process by which a person begins to acquire the physical, social and / or legal characteristics of the felt gender identity.

Transgender: An umbrella term usually referring to persons whose experienced or expressed gender does not conform to normative social expectations based on the gender they were assigned at birth.

Transsexual: A term often reserved for the subset of transgender individuals who desire to modify, or have modified, their bodies through hormones or surgery to be more congruent with their experienced gender.

"Trans": More recent umbrella terms being increasingly used to avoid distinguishing between transgender and transsexual individuals.

\section{Introduction}

The first reference to transsexuality in the medical literature, was made by the sexologist Friedrich Otto Westphal in 1930 [2]. He described it as "paranoid sexual metamorphosis" mainly influenced by Magnus Hirschfeld, who in 1910 spoke 
of the "psychic transsexual" as a form of "transvestic fetishism", given that a clear separation between the concepts of transvestism, transsexuality and homosexuality had not been established yet.

In 1949, the psychiatrist David Oliver Cauldwell coined the term "transsexual" for the first time, as it is used today. Almost two decades afterwards, the endocrinologist Harry Benjamin, in his book "The Transsexual Phenomenon" (1966), described his initial diagnosis of transsexuality, thus popularizing this concept.

In 1977, the term transsexualism became part of an international classification [3], the ICD-9; it was included, like paraphilias and sexual dysfunctions, within the "Sexual Deviations and Disorders" [4].

In 1980 [5], this same term appeared for the first time in the official nomenclature of the American Psychiatric Association (DSM-III), under the section "Sexual Identity Disorders" [6]. In the DSM-III, besides having reached puberty (otherwise it was referred to as "sexual identity disorder in childhood"), two requirements were needed to confirm the diagnosis: 1 ) Persistent malaise with regard to the anatomical sex itself; and 2) "A concern lasting at least two years, about how to get rid of the primary and secondary sexual characteristics, and how to achieve the sexual characteristics of the other sex".

Since its publication, many protests have arisen about this "pathologizing" term, a fact that was not enough to withdraw the concept of "disorder" in the subsequent publication of the ICD-10 (1992) [7] and the DSM-IV (1994) [8], which includes the qualification of Gender Identity Disorder, and Sexual Identity Disorder, respectively.

Currently, the ICD-11 [9] considers the term gender incongruence as the presence of a marked and persistent disagreement between the gender experienced by the person and the assigned sex. This often leads to a desire for "transition" to live and to be accepted as a person of the experienced gender. However, this classification will only apply after 2022. On the other hand, the DSM-V [10] considers gender dysphoria as a marked incongruence between the sex that one feels or expresses and the sex assigned at birth. In addition, unlike the ICD-11, it requires clinically significant discomfort or impairment in social, occupational, or other important functioning areas.

The prevalence of transsexualism is quite variable among the different studies, between $0.4 / 100.000$ and 23.6/100.000. A meta-analysis published in 2015 [11] collected data from 21 studies of similar methodology and analyzed the prevalence based on a demand for assistance. The results gave a prevalence of 6.8/100,000 MtF and 2.6/100,000 FtM. Our Madrid study, provided a prevalence rate of transsexualism of $1 / 3205$ $(31.2 / 100,000) \mathrm{MtF}$ and 1/7752 $(12.9 / 100,000)$ FtM [12].

In recent years, treatments improvement and the greater sociocultural acceptance of gender variability seem to be determining factors in the increase of transsexual people requesting health care [13], and even in the number of referred adolescents [14-16].
Nonetheless, many of these transgender or gender non-conforming people are still victims of prejudice, persecution and harassment. Many studies support the fact that the transgender population has significantly higher rates in terms of psychiatric comorbidity, with alarming figures for depression, anxiety, self-harm behaviors and suicidal tendencies $[16,17]$, as well as eating disorders or autism spectrum disorders, among others $[18,19]$. Furthermore, if we focus on a particularly vulnerable group, such as transgender adolescents, those events seem to gain even more force.

In recent years, the amount of trans adolescents attended in gender identity units has grown exponentially. This is linked to important epidemiological changes such as an early age of attendance, and a reversal in the ratio female/male. Up to now, in most studies the ratio in favor of trans female was evident, while recent studies show, (probably with increasing adolescent representation), that this proportion is equalizing and even reversing in favor of trans male [20,21].

The psychiatric comorbidity in these patients shows shocking figures $[22,23]$. Some studies demonstrate that $40-$ $45 \%$ of trans adolescents, have some type of psychiatric comorbidity [24]. Among them, the most frequent are depression, anxiety, self-harm episodes and suicide attempts [25]. Furthermore, in recent years we are noticing other events in the trans adolescent population, such as rapid onset gender dysphoria and desistence.

Rapid onset gender dysphoria refers to the sudden onset of symptoms related to gender dysphoria, which appears during puberty or even afterwards. Littman, in 2018 [26,27], suggested that the media influence or even a more permissive environment may be influencing this. Marchiano, et al. [14] even consider in some adolescents, the discomfort with their bodies as a determining factor.

Furthermore, other studies [28-30] suggest that up to $80 \%$ of transgender children and adolescents seem to give up, or in other words, they try to identify as cisgender when reaching an older age. We believe this is of the utmost importance, because the reversing desire is only innocuous if a treatment with non-reversible consequences has not been started. We refer to cross-hormonal therapy or gender affirmation surgeries; after this, it could even lead to a desire of detransition [31].

Many authors have responded to these worrisome figures, claiming that many of the children included in the study did not present diagnostic criteria for gender dysphoria, but rather did not comply with their gender.

Neither rapid onset gender dysphoria nor desistence are considered strictly psychiatric comorbidities.

\section{Research Hypothesis}

There are many and diverse factors that can influence this increased risk in the trans population of presenting various psychiatric comorbidities. Among the most relevant causes, we can consider the "non-acceptance" (normalization) of this condition, and the "minority stress" [32]. Furthermore, the psychiatric comorbidity in this population may be influenced 
by non-modifiable circumstances, such as the age of onset, the gender that the person identifies with, age of starting hormonal treatment or gender affirmation surgery [33].

On one side, we will try to investigate the epidemiological data regarding mental health in the transsexual population; on the other side, to look into their possible etiological factors, the distribution in different age groups or variation depending on the medical treatment.

\section{Appropriateness}

As it has been mentioned before, this population group has a high burden of psychopathological and psychiatric pathology. This justifies comprehensive investigation, not only to find etiological and epidemiological correlations with this high prevalence, but also to raise awareness and give tools to health professionals in general, and mental health professionals in particular, to be able to provide optimal care to this vulnerable sector of the population.

In recent years, the growing interest in offering quality care to this sector of the population has produced many papers that have shed new light on mental health in the transsexual population, particularly in the adolescent population.

All this highlights the need to revisit the recent literature in order to update the new evidence and probably, to focus in a special way on the growing number of adolescents who are part of this minority.

\section{Objectives}

The main objective of our paper is a bibliographic review of the literature published in the last five years (from 2015 to 2020) about mental health in the transsexual population. We will take in consideration not only the newness due to their more recent appearance (as they have not been included in previous reviews), but we will also take into account other variables, such as the impact that the treatment could have on these pathologies, the moment of appearance, or the age of the patients.

It will be of special relevance to review the psychiatric pathology in transsexual children and adolescents, as well as the rapidly onset gender dysphoria, desistance, detransition and regret.

Furthermore, we will try to understand the weight of the different etiological factors in the psychiatric vulnerability of this minority. This could help in a prevention strategy to lower the psychiatric pathology of the transsexual population and to be equated with that of the not transsexual population.

\section{Material and Methods}

\section{Selection criteria to narrow down the search}

Among the eligibility criteria used for this review, we included articles published from 2015 onwards, in English or Spanish, presenting quantitative data related to the epidemiology of different psychiatric comorbidities in a transgender population. On the one hand, several studies about different psychiatric comorbidities were included, as well as articles focused exclusively on prevalence of suicide or suicidal ideation, substance abuse, gaming addiction, depression and anxiety, eating disorders, autism spectrum disorders, dissociative disorders and non-suicidal self-harm attempts. Articles related to two phenomena, not strictly classified as psychiatric comorbidity, but which were of interest due to their relationship and involvement were also included: desistance and rapid onset gender dysphoria.

Articles that included both younger and older patients were included in the review, thus deciding on the greater weight that the child and adolescent population is taking in this context. Articles that studied diverse gender identities, such as non-binary or gender fluid, were also included, specifying them when addressing the results.

We specifically excluded papers addressing issues other than psychiatric pathology, such as quality of life, subjective perception of minority stress, non-psychiatric medical conditions, papers focusing exclusively on variables such as HIV positivity or the sexual orientation of the patients, and risky sexual behaviors.

\section{Information regarding search procedure and document selection}

An electronic search was conducted from June 2019 to February 2020 using MEDLINE / PubMed as the main database.

The keywords that were included to limit the search were (Transgender OR transsexual) AND (Mental health OR psychopathology OR psychiatric OR eating disorder OR autism OR suicide OR detransition OR depression).

In addition, a specific search was conducted including the term "Rapid Onset Gender Dysphoria", because due to its different nature it was not found in the previous search.

The range of articles was broadened by conducting an analysis of the bibliography included in previous reviews, always considering the above mentioned eligibility criteria.

Various articles were also accessed through Google Scholar.

\section{Search results and document selection}

We found 1,196 articles in the previously suggested databases. Of these, 717 were excluded after eliminating duplications and carrying out a first screening by reading the Title and Abstract, therefore 479 were initially selected, 12 were added by manual search, and after applying the eligibility criteria, 407 articles were excluded. Another 77 articles were also excluded because they were not original papers (meta-analyses, opinion articles, bibliographic reviews, etc.). We also excluded 137 articles because they did not show quantitative data that made reference to the epidemiology of the different psychiatric pathologies, or because they were qualitative studies that did not fit with our proposed review. Another 41 articles were excluded because they did not carry out a specific approach to psychiatric pathology or were subject to variables that could hinder the comparison with other results. In the same way, 152 articles were ignored because they had been published before 2015, a cut-off point that is 


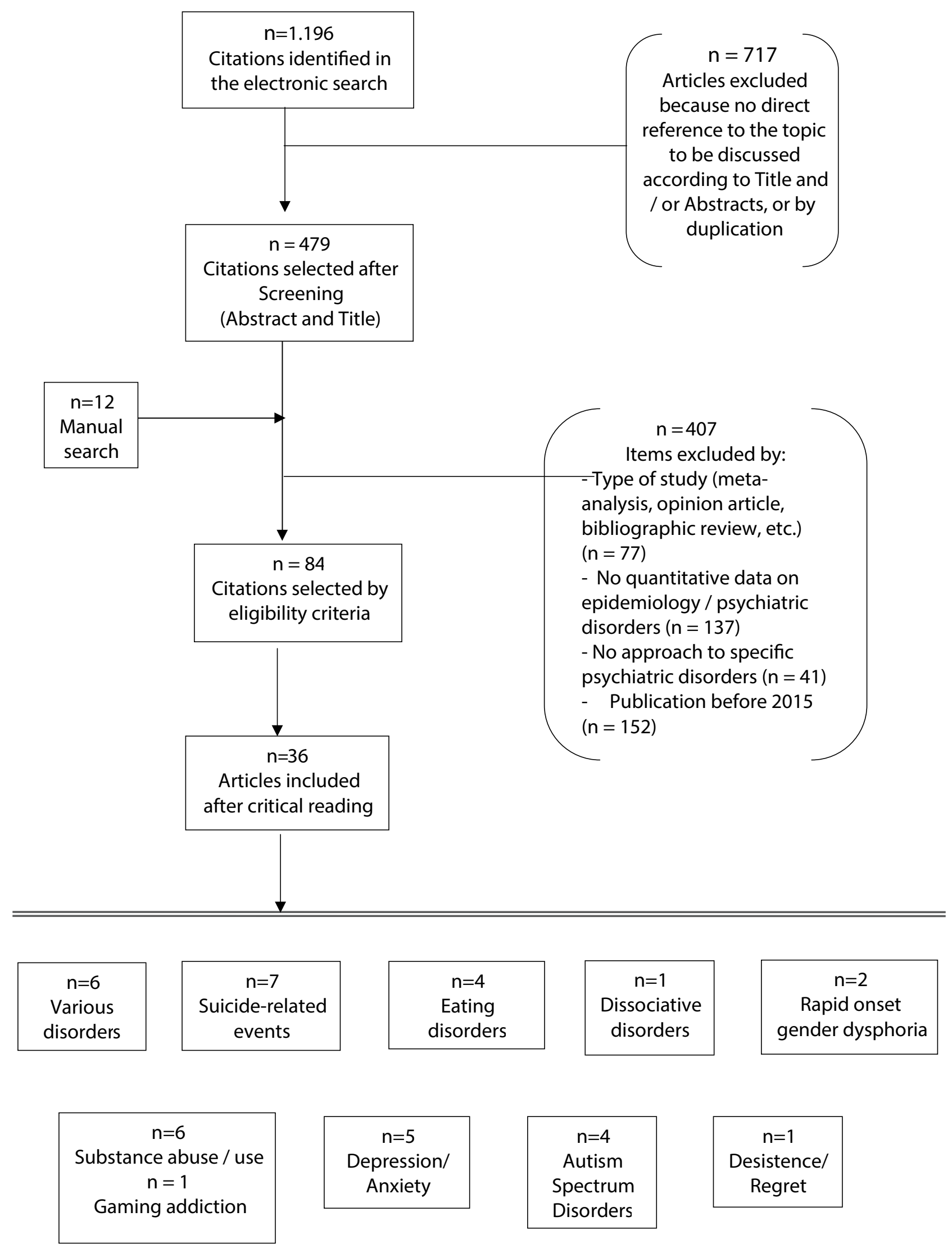

Figure 1: Flow chart of study. 
considered in the present work, due to the existence of relevant bibliographic reviews prior to that date (Figure 1 ).

\section{Description of the selected studies}

Cross-sectional studies: In total, 27 cross-sectional articles were included. We explain the basic characteristics of the same, with previous classification according to the psychiatric pathologies addressed.

\section{1) Various pathologies}

Among the cross-sectional articles, three of them [34-36] refer to different psychiatric pathologies, not focusing on the study of one in particular. In this way, depression, anxiety and post-traumatic stress disorder are represented in all of them. Substance abuse exclusively in Reisner, et al. [34], while suicide and self-harm behaviors are excluded in Cheung, et al. [35]. Autism spectrum disorders and eating disorders are specified in two of the studies $[35,36]$. Attention deficit hyperactivity disorder and obsessive-compulsive disorder in Cheung, et al., [35] and Peterson, et al. [36] is the only one that provides data on schizophrenia, conversion disorder, trichotillomania, and Tourette's syndrome.

All of them included male and female transsexuals, with the exception of Reisner, et al. [34], which only included trans female.

Regarding the age, Peterson, et al. [36] is the only one that includes the population from 12 years of age, with 16 being the cut-off point for the other two studies. Cheung, et al. [35] is the only one which included people over 29 years old.

\section{2) Substance abuse/Gaming addiction}

Six cross-sectional articles refer exclusively to substance abuse. All of them study the use of alcohol and cannabis, as well as other illicit drugs (cocaine, amphetamines, etc.). The use of certain non-prescribed medications such as amphetamines, analgesics (mainly opioids) is also included in all of them. Tobacco was included in five studies, but not in González, et al. [37].

Within the six studies cited, five of them included variables that are risk factors for substance misuse, such as social stigma, gender-related stress, previous victimization or the coexistence of other psychiatric pathologies such as depression or post-traumatic stress disorder. The only one that does not take into account any additional variable is De Pedro, et al. [38]. González, et al. [37] was the only one that was conducted in older than 18 years.

Three of them were carried out in minors [38-40], and two of them in adolescents and young people [41,42].

Three articles compared the prevalence between young transsexuals and non-transsexuals [38-40].

We also included a paper that studied the prevalence and degree of gaming addiction in the transsexual population and its associated factors [43].

\section{3) Suicide-related events}

Seven cross-sectional articles included events related with suicide. Four of them studied suicide attempt [44-47], five suicidal ideation [45-49] and one of them [47] included other concepts such as passive ideation of death or non-suicidal self-harm attempts. Claes, et al. [50] show the relationship between non-suicidal self-harm attempts with psychological factors and interpersonal problems, in transsexual people between 17 and 77 years old, and in Azeem, et al. [46] the prevalence of suicidal ideation in the trans adult population is related with the coexistence of depression. Three papers [4749] included minors in the sample, and in one [49] exclusively minors.

All papers excepting two $[46,47]$ considered different variables such as internalized transphobia, demographic and psychological variables or concomitant mental illnesses, and their relationship with the prevalence of the previously mentioned concepts.

\section{4) Depression/Anxiety}

Three articles address the contribution of different circumstances such as the social network, internalized heterosexism, stress or social support to the development of depression. Only one of them [51] also refers to generalized anxiety disorder.

Chodzen, et al. [52] included only minors, while Hoy-Ellis, et al. [53] was exclusively in elderly people. The remaining two included a wide range of ages, from minors to 79 years old.

\section{5) Eating disorders}

Another three studies focused primarily on eating disorders. Watson, et al. [54] included young subjects and took into account different protective and risk factors for the development of eating disorder behaviors. Avila, et al. [55] studied weight manipulations in young people as a purpose to affirm gender, and Diemer, et al. [18] included only adults to compare the prevalence of anorexia and bulimia nervosa in groups with different gender variants.

\section{6) Autism spectrum disorders}

Regarding autism spectrum disorders, two cross-sectional articles were included. Van der Miesen, et al. [56] studied different symptoms related to autism spectrum disorders in children and adolescents with and without gender dysphoria. Stagg \& Vincent [57] compared the prevalence of autistic disorder traits and undiagnosed autism among transgender, cisgender, and non-binary older people.

\section{7) Sudden onset gender dysphoria}

Due to the increasing interest in this phenomenon, a cross-sectional article and its correction were included $[26,27]$. They summarized the observations, experiences and perspectives of the parents of a group of transgender adolescents and young people on possible signs of appearance of abrupt gender dysphoria.

Longitudinal studies: Regarding longitudinal studies, we included 10 articles that will be classified like the previous one. 
Citation: Paz-Otero M, Becerra-Fernández A, Pérez-López G, et al. (2021) A 2020 Review of Mental Health Comorbidity in Gender Dysphoric and Gender Non-Conforming People. J Psychiatry Treat Res 3(1):44-55

\section{1) Various pathologies}

Three longitudinal articles are included in this category. In all of them, the prevalence of different psychiatric pathologies (depression and anxiety, suicide related events, eating disorders, substance abuse, autism spectrum disorders, etc.) were compared between the cisgender population and the transgender or gender nonconforming population.

Two of the studies $[22,58]$ carried out a retrospective and prospective cohort study, while Reisner, et al. [59] used a retrospective cohort, and Becerra-Culqui, et al. [22] was carried out in minors. Reisner, et al. [59] included a range of age between 12 and 29 years old, and Quinn, et al. [58] included a population from 3 to over 55, conducting a study on general health (including the mental health category) in the transsexual population.

\section{2) Dissociative disorders}

The study by Colizzi, et al. [60] developed a prospective cohort to assess the influence of hormonal treatment on the appearance of dissociative disorders in a transsexual population.

\section{3) Depression/Anxiety}

Two longitudinal case-control studies are included in our review $[61,62]$. Both were conducted in a sample of transgender children who had already transitioned and had good social and family support, the presence of depression, anxiety and similar self-esteem than a similar sample of cisgender children.

\section{4) Eating disorders}

One retrospective cohort study is included [63], comparing the presence of compensatory behaviors and the diagnosis of eating disorders in a university population between 18 and 26 years old, according to whether they were transgender, cisgender or sexual minorities.

\section{5) Autism spectrum disorder}

Two longitudinal case-control studies were included [64,65]. While in Nobili, et al. [64], traits related to autism spectrum disorder were compared in a large population aged 16 to 74, VanderLaan, et al. [65] analyzed in children under 12 years of age the possible mechanisms that could favor the co-occurrence of autism spectrum disorder and gender dysphoria.

\section{6) Desistance/Regret}

Through a large retrospective cohort, Wiepjes, et al. [31] analyzed not only the trend in terms of prevalence and treatment of trans people in recent decades, but also studied the percentage of desistance or regret after receiving treatment, as well as the aforementioned causes that justified it.

\section{Results}

We analyzed all the selected references according to the different groups of the psychiatric pathologies. We summarize the different studies included in this review, in Table 1, Table 2 and Table 3.

\section{1) Various pathologies}

The important prevalence of psychiatric comorbidity in the transsexual population is shown in the different studies, both cross-sectional and longitudinal, that addressed various psychiatric pathologies as a whole.

Most of the studies included adolescent and young trans people (under 30 years of age), showing a higher prevalence of psychopathology than in the cisgender population $[22,34,59]$. In up to $58-71 \%$ of the cases we could find a psychiatric diagnosis $[22,36]$, the most frequent pathologies being depression (with prevalence of up to 50\%) [38], self-harm $(8 \%)$ and anxiety (37.2\%) [22,36,59].

These pathologies have a prevalence two to three times higher than in the non-transgender population [34,36,59].

Two studies including an older population also concluded that depression (reaching 55.7\%) [35] and anxiety (43.5\%) [58] had a very high prevalence.

The youngest trans population group ( 3 to 9 years old), studied by Becerra-Culqui, et al. [22], shows a prevalence of $31 \%$ in some psychiatric diagnosis, the most frequent being Attention Deficit Disorder, followed by anxiety. However, from 10 to 17 years of age, this prevalence increases dramatically, reaching $71 \%$, with depression ranking first.

We can conclude that the prevalence of psychiatric comorbidity increases with age, and the adolescence being an especially vulnerable period.

The significant increase in the number of trans people requesting health care, as well as the increase in the prevalence of non-binary people [35] should encourage new studies. We should also pay special attention to trans male, because their incidence in health services is increasing exponentially, and also presenting at a younger age $[35,49]$.

\section{2) Substance use/Gaming addiction}

Only one study [37] referred to substance abuse and not to substance use. Furthermore, it was the only one including older population, with a mean of 32.6 years. It showed a high prevalence of substance abuse in the transsexual population $(40 \%)$, with cannabis in the first place $(24.4 \%)$, followed by alcohol $(21.5 \%)$, with equal prevalence in trans female and male. Other illegal drugs reach up to $11.6 \%$, being more frequent by trans male.

Studies about substance use in young people and adolescents ranked alcohol first, with a prevalence between $70 \%$ and $82 \%[39,42]$. Several studies coincide in placing cannabis in second position, closely followed by tobacco, but with a highly variable prevalence in both studies. The consumption of other drugs such as ecstasy, cocaine and non-prescribed analgesics and anxiolytics is also evident $[39,41,42]$.

While the prevalence of alcohol and cannabis in relation to the non-trans population is high (between 1.25-1.53: 1) $[39,42]$, this difference becomes greater when considering the use of ecstasy or cocaine, being up to 6 or 8 times higher [39]. 
Citation: Paz-Otero M, Becerra-Fernández A, Pérez-López G, et al. (2021) A 2020 Review of Mental Health Comorbidity in Gender Dysphoric and Gender Non-Conforming People. J Psychiatry Treat Res 3(1):44-55

Two studies included a younger trans population, De Pedro, et al. [38] with a sample above 12 years of age and Day, et al. [40] above 7. Substances consumption had a lower prevalence in all categories, but the distribution was similar. Despite this, the figures were still higher than in the non-trans population, and the consumption of other illegal drugs also presented higher proportions $[38,40]$ with trans boys and girls beginning to consume at an earlier age [40].

Regarding gaming addiction, Arcelus, et al. [43] published a prevalence of $62.9 \%$ of trans gamers, while only $0.7 \%$ were problematic cases. Furthermore, the presence of pathological gaming would have a lower prevalence in the trans population than in the cis one, with no difference between genders.

\section{3) Suicide-related events}

Within the events related to suicide, we would differentiate among suicide attempts per se, to suicidal ideation or passive ideas of death.

Firstly, regarding suicide attempts, three studies included samples between 14 and 30 years of age, with different prevalence. Chen, et al. [45] reported 16.1\%, while Kuper, et al. $32.3 \%$ [48] and Thorna, et al. 50.3\% [47]. Nonetheless, only $13.2 \%$ of the latter required medical attention.

Perez-Brumer, et al, with subjects with a mean age greater than 30 years, showed a prevalence of $32.4 \%$ [44].

In contrast, two studies that included samples from 12 years of age presented prevalences ranging between $17.2 \%$ [59] and 30.3\% [36].

Regarding the predominance of suicide attempts in either gender, there seems to be no agreement between studies, with two studies demonstrating a preponderance in trans male $[36,44]$ and three studies in trans female $[45,47,48]$.

Both Chen, et al. [45] and Thorna, et al. [47], showed a prevalence between 1.6 and 5.4 times higher than the nontrans population.

Regarding suicidal ideation, several studies analyzed the prevalence in people under 30 , showing quite different figures. Two studies published a prevalence of around $30 \%$ $[49,59]$; however Thorna, et al. [47], showed $84.8 \%$, while Kuper, et al. [48] published a worrying $95.5 \%$ in a young population with risk factors such as victimization and lack of support.

In people over 30 years of age, suicidal ideation reached a prevalence of $42.9 \%$, while in the population over fifty, it was $65.7 \%$ [46].

Trans females have a higher prevalence compared to trans males $[36,45,47,48]$ and this risk is also increased compared to that of the general population $[45,47,49]$.

Both suicide attempts and suicidal ideation seem to be especially present in adolescents and young adults, and the population over 50 is also particularly vulnerable $[36,46,48]$.

4) Self-harming non-suicidal attempts

Two studies addressed this topic. Claes, et al. [50], with a population with a mean age of 34.5 years, showed a preva- lence of $36.8 \%$ of self-harming non-suicidal attempts. Thorna, et al. [47], focused on adolescents between 14 and 18 years old, publishing a prevalence of $86.9 \%$.

Both concluded a predominance in trans males, as well as a positive association with age.

\section{5) Depression/Anxiety}

Both depression and anxiety have a high prevalence in young and adolescent trans populations.

In this group, the prevalence of depression ranged from $33 \%$ to $50.6 \%$ and anxiety had a wider range, between 26.7 and $63.3 \%[22,34,36,51,59]$.

On the other hand, three studies that included a population older than 30 years showed from $23.9 \%$ to $52 \%$ for depression $[51,53,58]$. Quinn, et al. [58] published $42 \%$ of anxiety in his group.

However, the most important difference we found in terms of depression and anxiety was in the studies that included younger people, from 3 to 14 years old. Anxiety ranged between $6 \%$ and $11.8 \%$ and depression was around $20 \%[49,61,62]$.

While in adolescents, young people and older adults the existence of this kind of psychopathology is between four and ten times higher than in the non-trans population $[38,40]$, if we focus on the child population, we find that anxiety is slightly increased with respect to cis boys and girls, while depression has the same proportion $[22,61,62]$.

\section{6) Eating disorders}

Eating behavior disorders are of obvious importance in this population group, due among others, to the potential use made of certain behaviors to achieve gender affirming purposes.

Studies that focused on trans adolescents and young people, between 13 and 26 years old, show that the presence of weight manipulations with gender affirming purposes reaches $65 \%$ [55]. However, if we take into account behaviors related to eating disorders, such as binge eating, fasting or vomiting and laxatives, this percentage drops between $33 \%$ and $50 \%$, the most frequent being fasting or binge eating, being more frequent in adolescent patients between 14 and 18 years of age than in young adults [54].

On the other hand, when we stick to the diagnosis of an eating disorder per se, Diemer, et al. [63] published $15.8 \%$ in the last year, which lower to $4.7 \%$ of anorexia or bulimia nervosa, in a study including a population older than 30 [18].

Although the prevalence of related behaviors and weight manipulations are much higher than the specific diagnosis of an eating disorder, the latter is still up to four times higher than in the non-trans population [18].

Adolescents, therefore, seem to be the most at risk group; it is remarkable that non-binary people, especially those assigned female at birth, have a greater prevalence $[18,55]$.

7) Autism spectrum disorders 
In recent years, several studies have been carried out, pointing to a higher prevalence of autism spectrum disorders in the trans population. Both Van der Miesen, et al. [56] and Stagg, et al. [57], showed similar results supporting these, while Nobili, et al. [64] published the same prevalence in trans and non-trans populations.

The prevalence of traits related to the autism spectrum disorders, was between $14.5 \%$, in a study carried out in minors [56], and $36.3 \%$, if we take into account the older population [64]. Nonetheless, the clinical diagnosis has somewhat lower figures, around 14\% [47].

Regarding the differences between genders, depending on the study, a higher prevalence was obtained in women [64] or an equal between both genders [56]. There is a higher prevalence in the non-binary population compared to the trans population, with non-binary people assigned female birth being overrepresented [57].

\section{8) Dissociative disorders}

Regarding dissociative disorders, the study carried out by Colizzi, et al. [60], published a prevalence of $29.6 \%$ of dissociative disorders in the trans population; this proportion was very high in comparison to the non-trans population, and showed no differences between genders.

\section{9) Rapid onset gender dysphoria}

In 2018, Littman published an $80.9 \%$ of rapid onset gender dysphoria [26]. The psychiatric comorbidity in these adolescents was $62.5 \%$, with anxiety (46.6\%) and depression $(39.4 \%)$, being the main diagnoses. Female assigned at birth were $82.8 \%$ and the average age at which they expressed their identification as trans was 15.2 years.

The vast majority did not present indicators of gender dysphoria in childhood or adolescence.

In Discussion, we will speak about their etiological and studied risk factors.

\section{0) Desistence}

Littman documented a $5.5 \%$ desistence [26], while Wiepjes, et al. [31] reported only 0.3-0.6\%.

In this way, this vast difference seems to be related with a greater presence of regret among those adolescents in whom gender dysphoria appeared abruptly.

Due to its consequences, it is evident the need to carry out more studies in order to clarify the possible influencing factors.

\section{Discussion}

The main objective of this paper is to investigate the mental health comorbidity in the transsexual population, through a systematic study of the literature in the last five years.

Most of the studies included here, mainly quantitative, seem to show a higher prevalence of psychiatric comorbidity than in the cisgender population. Nonetheless, it is difficult to link all the different results because their approach is quite different and the population sample can be quite varied.
An attempt has been made to combine the different ages in the various publications, and we found that transsexual adolescents and young adults seem to present greater psychiatric comorbidity than trans boys and girls or older adults.

Some studies collected the hormonal or surgical treatment received. However, when showing the results, many studies did not make a comparison of prevalence between the different groups. Likely, in the future further and more quality research will have to be conducted.

Among all the psychiatric comorbidities considered, the most frequent and relevant are depression, suicidal ideation and anxiety. This is important when approaching another objective that was raised in this review: The understanding of possible variables or factors that can act as facilitators or as protectors against psychopathology.

Firstly, if we look at the studies that reviewed depression and anxiety, similar results are shown in boys and girls who transitioned with social and family support in relation to the general population, showing only slight increases in anxiety $[60,61]$. In addition, the latter adds that self-esteem, apart from family support and the possibility of making a social transition were the main resilience factors that would explain these favorable results [61].

It is worth highlighting some factors that are seen in trans people much more frequently than in the general population. Among them, low self-esteem, poorer personal functioning, or significant dissatisfaction with one's own body [36,51]. In addition, anxiety and depression in this population seem to be increased by the significant internalized transphobia and the low congruence between appearance and referred gender [52].

These factors are the consequence of a lack of support and high perceived stress, items that were also presented as facilitators of psychopathology in this population [51,53].

Regarding gender affirming treatments, Witcomb, et al. [51] concluded that untreated transgender people have a higher risk of suffering from depression.

Secondly, the high prevalence of suicide ideation or attempts is evident, as well as its relationship with the aforementioned affective disorders. Thus, the existence of a previous psychiatric history (self-harm, attention in mental health services, the consumption of illicit drugs and depression as the main comorbidity) are predisposing factors of great relevance $[45,46,48,50]$.

Finally, it should not be forgotten that internalized and structural stigma, family conflicts, victimization and lack of social support are the main facilitators for the occurrence of suicide-related events [44,45,48-50].

Chen, et al. [45] reported an increase of suicidal ideation in the population that wants gender affirmation surgery. Nonetheless, hormonal treatment and gender affirmating surgery had not demonstrated any increase in the risk of suicide attempts.

To a lesser extent, but still highly represented, we find worrying rates of substance abuse and consumption, eating 
disorders, autism spectrum disorders and dissociative disorders in the transsexual population.

We found substance abuse and consumption as a significant issue in the trans population. Besides the excessive consumption of substances, also the minority stress, family instability, post-traumatic stress disorder and gender-related discrimination appear as decisive elements in the vulnerability of the trans community [37,40-42].

Within eating disorders, external stigma, understood as harassment or discrimination, is at the forefront as a potential risk factor for engaging in compensatory behaviors or weight manipulations for gender-affirming purposes [54]. Queries about sexual orientation also seem to have an association [18], while family, school and social support, a firm network of friends are protective factors for these pathologies.

Autism spectrum disorders, on the other hand, offer more controversial results in terms of their prevalence and their relationship to different circumstances. However, a study that linked high birth weight with gender dysphoria and eating disorders deserves to be highlighted, due to the novel association and the possibilities for future research [65].

Regarding dissociative disorders, trans people who received cross-hormonal treatment or gender affirmation surgery presented a lower incidence [60].

Despite not being part of psychopathology as such, in 2018 Littman [26] comprehensively addressed rapid onset gender dysphoria, stating that some agents such as family conflict, the circles of friendships in which most of the members are trans, or the gender variability increasingly represented in social networks and the media, could be favoring the growing influx of this manifestation. Littman's study established the need for further research, as it rose up a large amount of criticism: during their follow-up they had a $5.5 \%$ of withdrawal in their trans adolescents [58,59]. This percentage is quite high if compared with that established by Wiepjes, et al. [31], less than $0.5 \%$. Nonetheless, if we go back to two studies carried out in 2011 and 2013 [28,29], we can see up to $80 \%$ desistance; therefore, it is easy to understand the urgent need to continue longitudinal studies that address the disparities of previous articles.

Bearing in mind the significant heterogeneity in terms of methodology and eligibility criteria of the different studies and, therefore, the obstacles that this entails when generalizing the results to the whole trans population, we believe that this paper provides data that highlight the importance of conducting papers to improve investigation in this field.

On one hand, the fact of having reviewed articles limited to the last five years allow us to affirm that psychiatric comorbidity in the trans population continues to present with a very high prevalence, despite the advances that are being provided in terms of treatment and accessibility to health services.

On the other hand, the increasing acceptance by the society of trans affairs, would protect a priori against the psychopathology that in many cases affects this group.

All this leads us to think about the importance of the ex- ternal circumstances in triggering the manifestation of psychiatric pathology. We should be conscious of our responsibility, not only as health professionals, but also as members of a society that should ensure the well-being of those people who could be considered today a particularly vulnerable minority.

\section{Acknowledgments}

The authors are indebted to Dr. Joseph Hogg, partner at Abbey House Medical Centre, for his critical review, helpful comments and careful English revision.

\section{Declarations}

\section{Funding}

No funding available. All authors declare no support from any organization for the submitted work.

\section{Conflicts of interest/Competing interests}

The authors declare that they have no conflict of interest. No specific funding was received from any bodies in the public, commercial or not-for-profit sectors to carry out the work described in this article.

\section{Consent for publication}

Domingo Ly, the corresponding author, has the right to grant on behalf of all authors, and do grant on behalf of all authors, an exclusive licence, on a worldwide basis, to permit this article (if accepted), to be published in your journal.

\section{Declarations}

\section{Funding}

No funding available. All authors declare no support from any organisation for the submitted work.

\section{Conflicts of interest/Competing interests}

None. No benefits in any form had been received or will be received from a commercial party related directly or indirectly to the subject of this article.

\section{Consent for publication}

Domingo Ly, the corresponding author, has the right to grant on behalf of all authors, and do grant on behalf of all authors, an exclusive licence, on a worldwide basis, to permit this article (if accepted), to be published in your journal.

\section{Availability of data and material (data transpar- ency)}

All data are available on request

\section{References}

1. Byne W, Karasic DH, Coleman E, et al. (2018) Gender dysphoria in adults: An overview and primer for psychiatrists. Transgender Health 3: 57-A3.

2. Becerra-Fernández $A$, Rodríguez-Molina JM, Lucio-Pérez MJ, et al. (2010) Transexualidad y adolescencia. Rev Int Androl 8: 165171. 
3. Fernández Rodríguez F, García-Vega E (2011) Surgimiento, evolución y dificultades del diagnóstico de transexualismo. Rev Asoc Esp Neuropsiq 32: 103-119.

4. World Health Organization (1978) International classification of diseases. ( $9^{\text {th }}$ edn), Ginebra.

5. Mas Grau J (2017) Del transexualismo a la disforia de género en el DSM. Cambios terminológicos, misma esencia patologizante. Rev Int Sociol 75: e059.

6. Kendell RE (1980) Diagnostic and statistical manual of mental disorders. ( $3^{\text {rd }}$ edn), American Psychiatric Press, Washington, DC.

7. World Health Organization (1992) International classification of diseases. (10 $\mathrm{th}$ edn), Ginebra.

8. Samuel B Guze (1994) Diagnostic and statistical manual of mental disorders, $\left(4^{\text {th }}\right.$ edn). American Psychiatric Association. Washington, DC.

9. World Health Organization (2018) International classification of diseases. (11 ${ }^{\text {th }}$ edn), Ginebra.

10. American Psychiatric Association (2013) Diagnostic and statistical manual of mental disorders, $\left(5^{\text {th }}\right.$ edn). American Psychiatric Press, Washington, DC.

11. Arcelus J, Bouman WP, Van Den Noortgate W, et al. (2015) Systematic review and metaanalysis of prevalence studies in transsexualism. European Psychiatry 30: 807-815.

12. Becerra-Fernández A, Rodríguez-Molina JM, Asenjo-Araque $\mathrm{N}$ et al. (2017) Prevalence, incidence, and sex ratio of transsexualism in the autonomous region of Madrid (Spain) according to healthcare demand. Arch Sex Behav 46: 1307-1312.

13. Becerra-Fernández A (2003) Transexualidad. La búsqueda de una identidad. ( $1^{\text {st }}$ edn), Ediciones Díaz de Santos, Madrid.

14. Marchiano L (2017) Outbreak: On transgender teens and psychic epidemics. Psychol Perspect 60: 345-366.

15. Spivey LA, Edwards-Leeper $L$ (2019) Future directions in affirmative psychological interventions with transgender children and adolescents. J Clin Child Adolesc Psychol 48: 343-356.

16. Shumer DE, Nokoff NJ, Spack NP (2016) Advances in the care of transgender children and adolescents. Adv Pediatr 63: 79-102.

17. Zucker KJ (2019) Adolescents with gender dysphoria: Reflections on some contemporary clinical and research issues. Arch Sex Behav 48: 1983-1992.

18. Diemer EW, Hughto JMW, Gordon AR, et al. (2018) Beyond the binary: Differences in eating disorder prevalence by gender identity in a transgender sample. Transgend Health 3: 17-23.

19. Van der Miesen AIR, Cohen-Kettenis PT, de Vries ALC (2018) Is there a link between gender dysphoria and autism spectrum disorder? J Am Acad Child Adolesc Psychiatry 57: 884-885.

20. de Graaf NM, Giovanardi G, Zitz C, et al. (2018) Sex ratio in children and adolescents referred to the gender identity development service in the UK (2009-2016). Arch Sexual Behav 47: 13011304.

21. Kreher J (2016) Do youth transgender diagnoses put would-be gay, lesbian, and bisexual adults at risk for unnecessary medical intervention? The homoarchy.

22. Becerra-Culqui TA, Liu Y, Nash R, et al. (2018) Mental health of transgender and gender nonconforming youth compared with their peers. Pediatrics 141: e20173845.
23. Kuper LE, Mathews S, Lau M (2019) Baseline mental health and psychosocial functioning of transgender adolescents seeking gender-affirming hormone therapy. J Dev Behav Pediatr 40: 589596.

24. Kaltiala-Heino R, Sumia M, Tyolajarvi M, et al. (2015) Two years of gender identity service for minors: Overrepresentation of natal girls with severe problems in adolescent development. Child Adolesc Psychiatry Ment Health 9: 1-9.

25. Kaltiala-Heino R, Bergman H, Tyolajarvi M, et al. (2018) Gender dysphoria in adolescence: Current perspectives. Adoles Health Med Ther 9: 31-41.

26. Littman L (2018) Parent reports of adolescents and young adults perceived to show signs of a rapid onset of gender dysphoria. PLos One 13: e0202330.

27. Littman L (2019) Correction: Parent reports of adolescents and young adults perceived to show signs of a rapid onset of gender dysphoria. PLos One 14: e0214157.

28. Steensma TD, McGuire JK, Kreukels BPC, et al. (2013) Factors associated with desistence and persistence of childhood gender dysphoria: A quantitative follow-up study. J Am Acad of Child Adolesc Psychiatry 53: 582-590.

29. Steensma TD, Biemond R, de Boer F, et al. (2011) Desisting and persisting gender dysphoria after childhood: A qualitative follow-up study. Clin Child Psychol Psychiatry 16: 499-516.

30. Singh D (2012) A follow-up study of boys with gender identity disorder. University of Toronto.

31. Wiepjes CM, Nota NM, de Blok CJM, et al. (2018) The Amsterdam Cohort of Gender Dysphoria Study (1972-2015): Trends in prevalence, treatment, and regrets. J Sex Med 15: 582- 590.

32. Hatzenbuehler ML, Pachankis JE (2016) Stigma and minority stress as social determinants of health among lesbian, gay, bisexual, and transgender Youth. Research evidence and clinical implications. Pediatr Clin North Am 63: 985-997.

33. Allen LR, Watson LB, Egan AM, et al. (2019) Well-being and suicidality among transgender youth after gender-affirming hormones. Clin Prac Pediatr Psychology 7: 302-311.

34. Reisner SL, Biello KB, Hughto JMW, et al. (2016) Psychiatric diagnoses and comorbidities in a diverse, multicity cohort of young transgender women: baseline findings from project life skills. JAMA Pediatr 170: 481-486.

35. Cheung AS, Ooi O, Leemaqz S, et al. (2018) Sociodemographic and clinical characteristics of transgender adults in Australia. Transgend Health 3: 229-238.

36. Peterson CM, Matthews A, Copps-Smith E, et al. (2017) Suicidality, self-harm, and body dissatisfaction in transgender adolescents and emerging adults with gender dysphoria. Suicide Life Threat Behav 47: 475-482.

37. Gonzalez C, Miner MH, Bockting WO (2017) An examination of demographic characteristics, components of sexuality, and minority stress as predictors of cannabis, excessive alcohol, and illicit/nonprescription drug use among transgender people in the United States. J Prim Prev 38: 419-445.

38. De Pedro KT, Gilreath TD, Jackson C, et al. (2017) Substance use among transgender students in California public middle and high schools. J Sch Health 87: 303-309.

39. Johns MM, Lowry R, Andrzejewski J, et al. (2019) Transgender identity and experiences of violence victimization, substance 
use, suicide risk, and sexual risk behaviors among high school students - 19 states and large urban school districts, 2017. MMWR Morb Mortal Wkly Rep 68: 67-71.

40. Day JK, Fish JN, Perez-Brumer A, et al. (2017) Transgender youth substance use disparities: Results from a population-based sample. J Adolesc Health 61: 729-735.

41. Fontanari AMV, Pase PF, Churchill S, et al. (2019) Dealing with gender-related and general stress: Substance use among Brazilian transgender youth. Addict Behav Rep 9: 100166.

42. Rowe C, Santos GM, McFarland W, et al. (2015) Prevalence and correlates of substance use among trans*female youth ages 16 24 years in the San Francisco Bay Area. Drug Alcohol Depend 147: $160-166$.

43. Arcelus J, Bouman WP, Jones BA, et al. (2017) Video gaming and gaming addiction in transgender people: An exploratory study. $J$ Behav Addict 6: 21-29.

44. Perez-Brumer A, Hatzenbuehler ML, Oldenburg CE, et al. (2015) Individual- and structural- level risk factors for suicide attempts among transgender adults. Behav Med 41: 164-171.

45. Chen R, Zhu X, Wright L, et al. (2019) Suicidal ideation and attempted suicide amongst Chinese transgender persons: National population study. J Affect Disord 245: 1126-1134.

46. Azeem R, Zubair UB, Jalil A, et al. (2019) Prevalence of suicide ideation and its relationship with depression among transgender population. J Coll Physicians Surg Pak 29: 349-352.

47. Thorna BC, Salk RH, Choukas-Bradley S, et al. (2019) Suicidality disparities between transgender and cisgender adolescents. Pediatrics 144: e20191183.

48. Kuper LE, Adams N, Mustanski BS (2018) Exploring cross-sectional predictors of suicide ideation, attempt, and risk in a large online sample of transgender and gender nonconforming youth and young adults. LGBT Health 5: 391-400.

49. Perez-Brumer A, Day JK, Russell ST, et al. (2017) Prevalence and correlates of suicidal ideation among transgender youth in California: Findings from a representative, population-based sample of high school students. J Am Acad Child Adolesc Psychiatry 56: 739-746.

50. Claes L, Bouman WP, Witcomb G, et al. (2015) Non-suicidal self-injury in trans people: Associations with psychological symptoms, victimization, interpersonal functioning, and perceived social support. J Sex Med 12: 168-179.

51. Witcomb GL, Bouman WP, Claes L, et al. (2018) Levels of depression in transgender people and its predictors: Results of a large matched control study with transgender people accessing clinical services. J Affect Disord 235: 308-315.
52. Chodzem G, Hidalgo MA, Chen D, et al. (2019) Minority stress factors associated with depression and anxiety among transgender and gender-nonconforming youth. J Adolesc Health 64: 467-471.

53. Hoy-Ellis CP, Fredriksen-Goldsen KI (2017) Depression among transgender older adults: General and minority stress. Am J Community Psychol 59: 295-305.

54. Watson RJ, Veale JF, Saewyc EM (2017) Disordered eating behaviors among transgender youth: Probability profiles from risk and protective factors. Int J Eat Disord 50: 515-522.

55. Avila JT, Golden NH, Aye T (2019) Eating disorder screening in transgender youth. J Adolesc Health 65: 815-817.

56. van der Miesen AIR, de Vries ALC, Steensma TD, et al. (2018) Autistic symptoms in children and adolescents with gender dysphoria. J Autism Dev Disord 48: 1537-1548.

57. Stagg SD, Vincent J (2019) Autistic traits in individuals self-defining as transgender or non-binary. Eur Psychiatry 61: 17-22.

58. Quinn VP, Nash R, Hunkeler E, et al. Cohort profile: Study of transition, outcomes and gender (STRONG) to assess health status of transgender people. BMJ Open 7: e018121.

59. Reisner SL, Vetters R, Leclerc M, et al. (2015) Mental health of transgender youth in care at an adolescent urban community health center: a Matched retrospective cohort study. J Adolesc Health 56: 274-279.

60. Colizzi M, Costa R, Todarello O (2015) Dissociative symptoms in individuals with gender dysphoria: Is the elevated prevalence real? Psychiatry Res 226: 173-180.

61. Olson KR, Durwood L, DeMeules M, et al. (2016) Mental health of transgender children who are supported in their identities. Pediatrics 137: e20153223.

62. Durwood L, McLaughlin KA, Olson KR (2017) Mental health and self-worth in socially transitioned transgender youth. J Am Acad Child Adolesc Psychiatry 56: 116-123.

63. Diemer EW, Grant JD, Munn-Chernoff MA, et al. (2015) Gender identity, sexual orientation and eating-related pathology in a national sample of college students. J Adolesc Health 57: 144-149.

64. Nobili A, Glazebrook C, Bouman WP, et al. (2018) Autistic traits in treatment-seeking transgender adults. J Autism Dev Disord 48: 3984-3994.

65. VanderLaan DP, Hughes SK, Leef J, et al. (2015) Autism spectrum disorder risk factors and autistic traits in gender dysphoric children. J Autism Dev Disord 45: 1742-1750.

DOI: $10.36959 / 784 / 425$

Copyright: (C) 2021 Paz-Otero M, et al. This is an open-access article distributed under the terms of the Creative Commons Attribution License, which permits unrestricted use, distribution, and reproduction in any medium, provided the original author and source are credited. 